03

\title{
Влияние уширения газового ресивера на баллистические характеристики одноступенчатых установок на сжатом газе
}

\author{
(C) Н.В. Быков, ${ }^{1,2}$ И.Е. Шестаков ${ }^{1}$ \\ ${ }^{1}$ Московский государственный технический университет им. Н.Э. Баумана, \\ 105005 Москва, Россия \\ ${ }^{2}$ Федеральный исследовательский центр химической физики им. Н.Н. Семенова РАН, \\ Москва, Россия \\ e-mail: bykov@bmstu.ru, ily.shestako@yandex.ru
}

Поступило в Редакцию 24 июля 2019 г.

В окончательной редакции 24 июля 2019 г.

Принято к публикации 16 сентября 2019 г.

Исследовано влияние степени уширения газового ресивера на выходную скорость тела при разгоне его в стволе сжатым газом. Моделирование проведено в рамках квазиодномерной и двумерной осесимметричной газодинамических моделей. Модели валидированы на экспериментальных результатах. Показано, что использование более широкого ресивера с тем же объемом и начальным давлением позволяет увеличить КПД на 25\%. Продемонстрировано существенное влияние сжатия воздуха перед легким снарядом при сверхзвуковых режимах на его скорость. Учет сжатия воздуха перед снарядом проведен с использованием приближенной формулы, что показало хорошие результаты по сравнению с двумерной моделью. Выявлен эффект ускорения легкого снаряда из-за выталкивания столба воздуха через дульный срез.

Ключевые слова: газовые пушки, газодинамический разгон тел, уширение камеры, методы ускорения тел, баллистические установки.

DOI: 10.21883/JTF.2020.03.48918.278-19

\section{Введение}

Способ разгона тел в стволе за счет расширения сжатого газа (одноступенчатые газовые пушки) широко востребован в лабораторных исследованиях динамических свойств материалов [1-3], а также при испытаниях конструкций на ударную прочность [4]. Схема пневматического разгона используется и в практических приложениях, например, в спортивном оружии [5] и спасательных линеметах [6].

Важными качествами установок на сжатом газе являются простота конструкции, относительно низкие уровни рабочего давления, отсутствие загрязнения внутренних поверхностей продуктами сгорания, а также высокая воспроизводимость выходных скоростей (в отличие, например, от пороховых пушек). Эти установки незаменимы, если по каким-то причинам в исследованиях нельзя использовать взрывчатые вещества и пороха.

Максимальные экспериментально зафиксированные значения дульной скорости при использовании гелия в качестве рабочего газа составили $1734 \mathrm{~m} / \mathrm{s}$ для тела массой $m=227 \mathrm{~g}$ в стволе калибром $d=62.74 \mathrm{~mm}$ при давлении в ресивере $34.5 \mathrm{MPa}$ [7], а также $1620 \mathrm{~m} / \mathrm{s}$ для ствола калибром $44 \mathrm{~mm}$ при массе тела $50 \mathrm{~g}$ и давлении в ресивере $45.6 \mathrm{MPa}$ [8]. Для воздуха и азота максимально зафиксированные в экспериментах скорости составляют 500 и $650 \mathrm{~m} / \mathrm{s}$ соответственно $[8,9]$. На рис. 1 показана зависимость максимально достижимых скоростей в известных экспериментах на одноступенчатых газовых пушках по результатам анализа источников [7-21] от отношения давления в камере $p_{0}$ к коэффициенту массы снаряда $c_{q}=m / d^{3}$, традиционно измеряемому в $\mathrm{g} / \mathrm{cm}^{3}$.
C точки зрения расчета режимов, а также конструирования газовых лабораторных установок, важнейшим вопросом является адекватное моделирование физических процессов, позволяющее предсказывать результаты эксперимента с высокой степенью точности. В настоящее время в расчетах преобладает использование приближенных аналитических формул и одномерных газодинамических моделей совершенного газа. Анализ приближенных одномерных моделей, а также численного решения полной системы одномерных уравнений приведен в работах [22,23]. К двумерному моделированию прибегают достаточно редко. Так, например, в работе [24] на примере частной задачи проанализировано влияние вязкости на процесс пневматического разгона поршня в стволе, при этом истечение из ресивера рассчитывалось в квазистационарном приближении, а в работе [25] проведено сравнение результатов двумерных и одномерных расчетов, которое в принципе показало удовлетворительное согласие обоих, однако массы метаемых тел варьировались в слишком узких диапазонах. Влияние коволюма газов было рассмотрено в работе [26].

В то же время надо заметить, что реальным баллистическим установкам присущи некоторые особенности, которые традиционными решениями, как правило, не учитываются. Среди них можно выделить как минимум три, существенно влияющие на результат функционирования установки.

Первая особенность заключается в использовании более широких по сравнению со стволом ресиверов (камер). Эта особенность формирует условия истечения газа из ресивера в ствол, отличные от приближения 


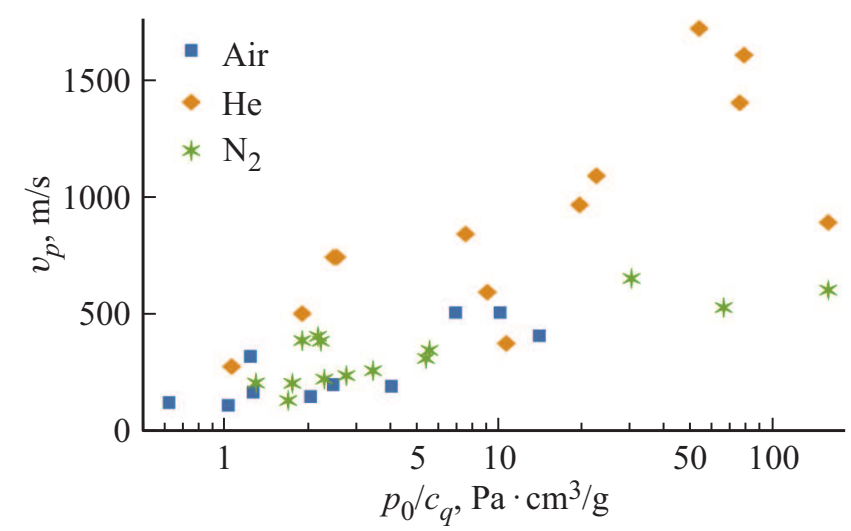

Рис. 1. Достигаемые экспериментально скорости метаемых тел на одноступенчатых газовых установках.

камеры приведенного диаметра (т.е. с диаметром, равным диаметру ствола). Для того чтобы ресивер такого диаметра имел эквивалентный объем, вводится его приведенная длина, в результате чего камера существенно удлиняется. Такое изменение геометрии существенно влияет на волновые процессы, поскольку время прохождения волны по камере прямо пропорционально ее длине. Кроме того, в уширенных камерах волны должны интенсивнее рассеиваться. Использование ресивера того же диаметра, что и ствол, встречается в практике, но крайне редко [7].

Вторая особенность заключается в сжатии воздуха перед метаемым телом, которое особенно сильно проявляется на сверхзвуковых скоростях движения, а также в ускоренном истечении воздуха из ствола при подходе воздушной ударной волны, создаваемой перед снарядом, к дульному срезу. Это не относится к вакуумированным трассам, которые при использовании газовых пушек встречаются не очень часто в силу относительно малых скоростей вылета тел.

Наконец, третья особенность заключается в способе открытия ресивера. Например, известно, что при использовании быстродействующих клапанов, газ из ресивера поступает в ствол медленнее, чем при использовании разрушающихся диафрагм, что может существенно влиять на скорость вылета тела [6]. В настоящей работе влияние этого фактора не рассматривается.

Целью настоящей работы является анализ влияния первых двух факторов на физические процессы внутри установки и выходную скорость метаемого тела. При этом следует отметить одну существенную особенность разгона поршня сжатым газом. Известно, что основным параметром, от которого зависит физический процесс разгона, является отношение массы снаряда (поршня) $m$ к массе разгоняющего его газа $M$, которое обозначим $\mu=m / M[22]$ В соответствии с этим имеются два предельных режима. Первый режим реализуется при $\mu \gg 1$, что соответствует тяжелому по сравнению с массой газа поршню. Для этого случая влияние волновых эффектов в газе несущественно. Во втором случае $\mu \ll 1$, поршень существенно легче разгоняющего его газа, и волновое движение последнего выходит на первый план. Исходя из этого, необходимо исследовать влияние тех или иных факторов в зависимости от реализующегося режима разгона, т. е. при различных $\mu$.

\section{Методы математического моделирования}

Для описания движения газа в баллистической установке в настоящей работе используются две модели квазиодномерная и осесимметричная.

Система квазиодномерных газодинамических уравнений имеет вид

$$
\frac{\partial(\mathbf{q} S)}{\partial t}+\frac{\partial(\mathbf{f} S)}{\partial x}=\mathbf{h}
$$

где

$$
\mathbf{q}=\left(\begin{array}{c}
\rho \\
\rho v \\
\rho E
\end{array}\right) ; \quad \mathbf{f}=\left(\begin{array}{c}
\rho v \\
\rho v^{2}+p \\
\rho v H
\end{array}\right) ; \quad \mathbf{h}=\left(\begin{array}{c}
0 \\
p \frac{\partial S}{\partial x} \\
0
\end{array}\right)
$$

Здесь $\rho, v, p, E, H$ - плотность, скорость, давление, полная энергия и полная энтальпия единицы массы газа соответственно, $S$ - площадь поперечного сечения трубы, $t$ - время, $x$ - пространственная координата.

Система (1) дополняется уравнением состояния идеального газа $p=(\gamma-1) \rho \varepsilon$, где $\gamma-$ показатель адиабаты рабочего газа, $\varepsilon$ - внутренняя энергия единицы массы газа, и уравнением движения метаемого тела (поршня)

$$
m \frac{d v_{p}}{d t}=\left(p_{p}-p_{a}\right) S,
$$

где $m$ - масса поршня, $v_{p}$ - скорость поршня, $p_{p}$ давление на поршень со стороны разгоняющего его газа, $p_{a}$ - давление на поршень со стороны внешней среды перед поршнем.

На левой границе расчетной области, соответствующей задней стенке ресивера, ставится граничное условие непротекания, на правой границе (подвижный поршень, $\left.x=x_{p}\right)$ - равенство скоростей газа и поршня, определяемой из (2).

Численное решение системы (1) осуществляется с помощью двухшаговой схемы „предиктор-корректор“ на подвижной сетке, правая граница которой отслеживает положение поршня [27]:

$$
\begin{aligned}
(\mathbf{q} W)_{i+1 / 2}^{n+1 / 2}= & (\mathbf{q} W)_{i+1 / 2}^{n}-\left(\tau^{n} / 2\right)\left[(\mathbf{f} S)_{i+1}^{n}-(\mathbf{f} S)_{i}^{n}\right. \\
& \left.+\mathbf{h}_{i+1 / 2}^{n} \Delta x_{i+1 / 2}^{n}\right], \\
(\mathbf{q} W)_{i+1 / 2}^{n+1}= & (\mathbf{q} W)_{i+1 / 2}^{n}-\tau^{n}\left[(\mathbf{f} S)_{i+1}^{n+1 / 2}-(\mathbf{f} S)_{i}^{n+1 / 2}\right. \\
& \left.+\mathbf{h}_{i+1 / 2}^{n+1 / 2} \Delta x_{i+1 / 2}^{n+1 / 2}\right],
\end{aligned}
$$




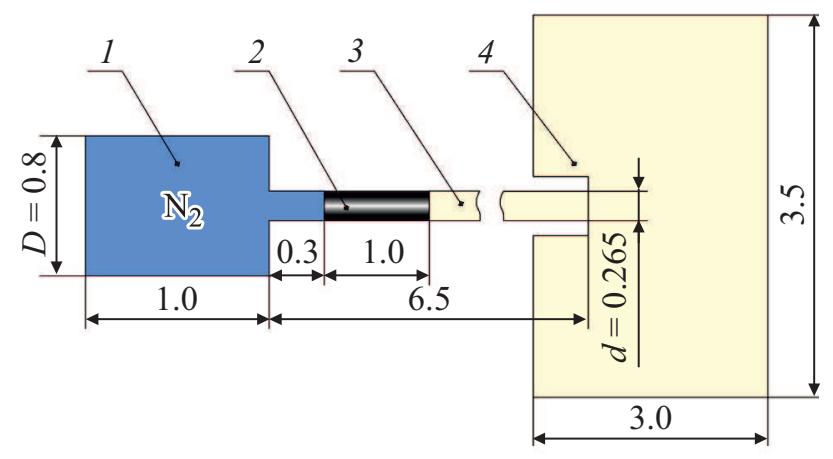

Рис. 2. Расчетная схема задачи (все размеры даны в метрах): 1 - ресивер, 2 - снаряд (поршень), 3 - баллистический ствол, 4 - внешнее пространство.

Таблица 1. Результаты сравнения расчетных и экспериментальных значений выходной скорости

\begin{tabular}{c|c|c|c|c|c}
\hline$p_{0}, \mathrm{MPa}$ & $m, \mathrm{~kg}$ & $\mu$ & $v_{p, \text { exp }}, \mathrm{m} / \mathrm{s}$ & $v_{p, 1 D}, \mathrm{~m} / \mathrm{s}$ & $v_{p, 2 D}, \mathrm{~m} / \mathrm{s}$ \\
\hline 4.0 & 27.0 & 1.10 & 231 & 235 & 238 \\
4.0 & 32.5 & 1.32 & 216 & 217 & 215 \\
4.0 & 42.5 & 1.72 & 199 & 194 & 192 \\
5.0 & 27.0 & 0.87 & 252 & 258 & 254 \\
7.8 & 27.0 & 0.56 & 305 & 310 & 292
\end{tabular}

Здесь индексы $n$ и $i$ отвечают временным и координатным узлам разностной сетки соответственно; $\tau_{n}-$ шаг по времени; $\Delta x_{i+1 / 2}^{n}$ - шаг по координате; $W_{i+1 / 2}^{n}-$ объем ячейки, который вычисляется по формуле для объема усеченного конуса. Потоки на границах ячеек определяются модифицированным методом AUSM+ аналогично работе [22].

Для моделирования течений газа в баллистической установке в осесимметричной постановке явным методом решалась двумерная осесимметричная задача в пакете ANSYS Fluent. Использовалась модель совершенного невязкого газа, потоки на границах ячеек рассчитывались с использованием схемы AUSM. Для учета движения снаряда в процессе выстрела использовалась подвижная сетка. Модель замыкалась уравнением движения поршня, реализованным с помощью пользовательской функции (user defined function, UDF). Для учета сжатия воздуха перед снарядом вводилась отдельная расчетная область, включающая ствол и внешнее пространство у дульного среза. Расчетная схема задачи изображена на рис. 2. За основу была взята геометрия баллистической установки, для которой известны результаты экспериментальных измерений скоростей $[12,25]$. Такой подход позволяет провести достаточно простую валидацию используемых моделей на экспериментальных данных. Рабочим газом является азот при температуре $283 \mathrm{~K}$, окружающий воздух имеет температуру $300 \mathrm{~K}$.

В табл. 1 приведены значения дульной скорости снарядов различных масс при варьируемом начальном дав- лении в резервуаре, полученные с помощью квазиодномерного расчета $\left(v_{p, 1 D}\right)$, осесимметричного расчета $\left(v_{p, 2 D}\right)$ и экспериментально $\left(v_{p, \exp }\right)[12]$. В одномерных расчетах противодавление со стороны воздуха перед снарядом учитывалось введением постоянного давления $p_{a}=0.1 \mathrm{MPa}$ в уравнение (2). Также для уменьшения градиента площади поперечного сечения в одномерных расчетах использовался сглаженный переход между камерой и стволом при сохранении объема камеры. Максимальная погрешность расчета относительно экспериментальных данных составила $4.3 \%$. Средняя погрешность для пяти вычислений не превышает $2.5 \%$.

\section{Влияние уширения камеры на выходную скорость метаемого тела}

Для оценки влияния уширения камеры на выходную скорость снаряда введем степень уширения камеры, равную отношению диаметра камеры к диаметру (калибру) баллистического ствола: $\delta=D / d$. Были рассмотрены три варианта степени уширения: $\delta=1(D=0.265 \mathrm{~m})$, $\delta=3(D=0.8 \mathrm{~m})$ и $\delta=6(D=1.6 \mathrm{~m})$. Остальная геометрия соответствует рис. 1. Для каждого из этих случаев были рассмотрены три различных массы метаемого тела, отвечающие значениям $\mu=0.1 \quad(m=3.089 \mathrm{~kg})$, $\mu=1(m=30.89 \mathrm{~kg})$ и $\mu=10(m=308.9 \mathrm{~kg})$. Давление азота в резервуаре для всех случаев принималось равным $p_{0}=5 \mathrm{MPa}$, начальная температура азота $283 \mathrm{~K}$, температура окружающего воздуха $300 \mathrm{~K}$.

Графики зависимостей скорости снаряда от его координаты для этих девяти случаев приведены на рис. 3 .

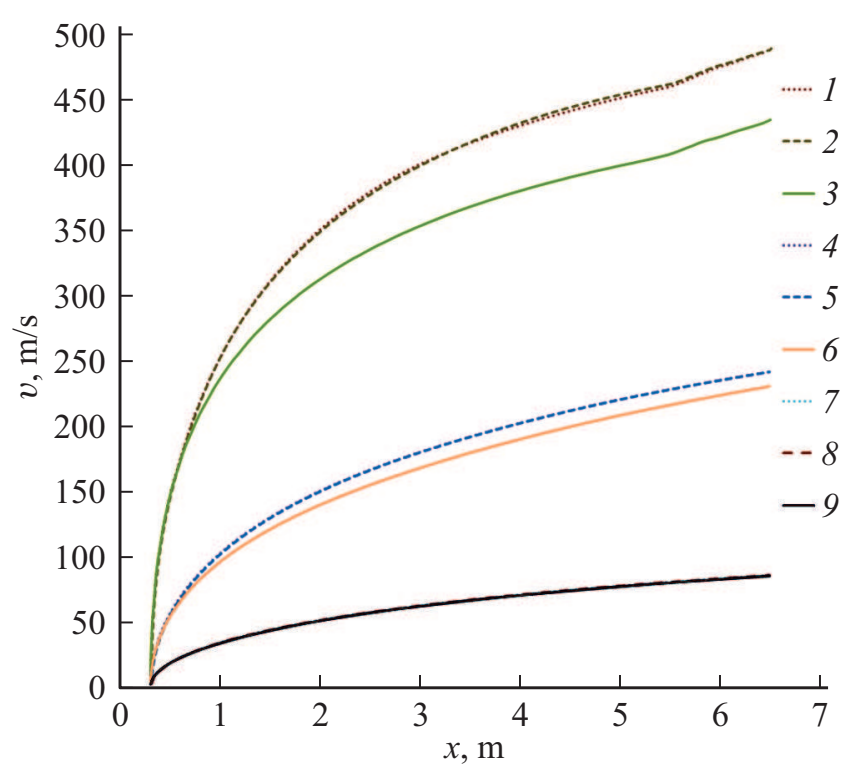

Рис. 3. Зависимости скорости снаряда от его координаты для различных степеней уширения камеры $(\delta)$ и относительных масс снарядов $(\mu)$. Кривые $1-3-\mu=0.1 ; 4-6-\mu=1$; $7-9-\mu=10 ; 1,4,7-\delta=6,2,5 ; 8-\delta=3 ; 3,6,9-\delta=1$. 

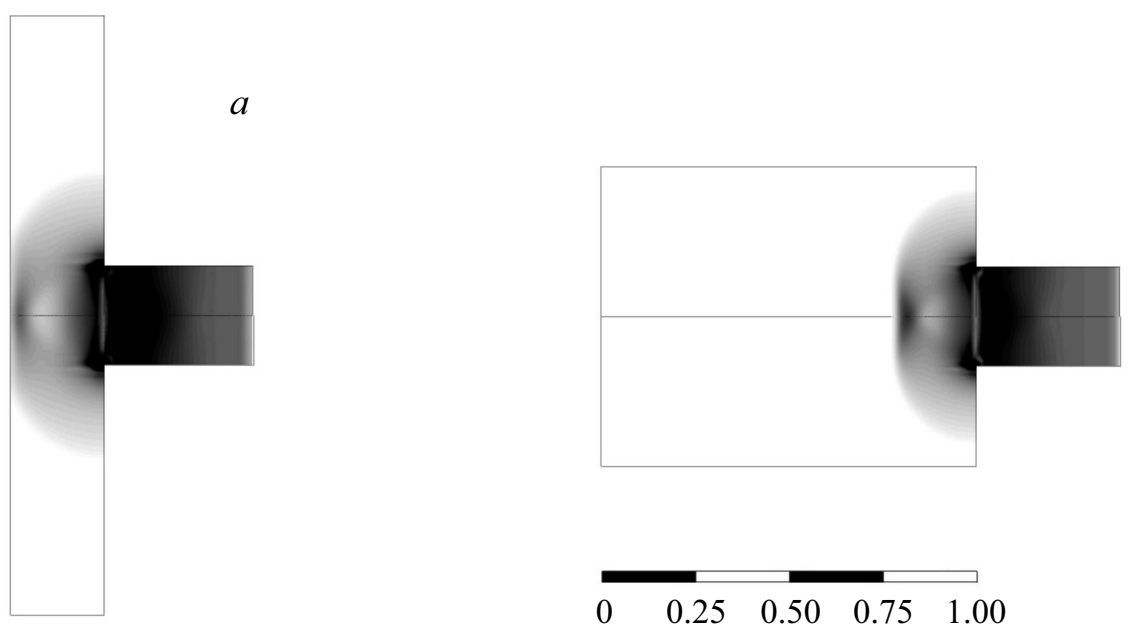

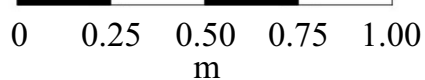

C

Рис. 4. Градиент давления для случая $\mu=0.1$ в момент времени $t=1.5 \mathrm{~ms}: a-\delta=6 ; b-\delta=3 ; c-\delta=1$.

Полученные распределения показывают, что для одинаковых начальных давлений в ресивере выходная скорость метаемого тела при $\delta=3$ выше, чем при $\delta=1$. В свою очередь, при $\delta=6$ существенных различий со случаем $\delta=3$ не наблюдается. Самая большая разница между уширенным и равным диаметру ствола ресивером соответствует случаю $\mu=0.1$ (кривые 1-3). В этом случае длина приведенной камеры эквивалентного объема превышает $9 \mathrm{~m}$, поэтому волна не успевает догнать легкий снаряд и реализуется режим вылета без влияния отраженной волны, который описывается точным решением [22,23]. Из этого следует, что при одинаковой массе и объеме газа уширение ресивера позволяет использовать газ более эффективно с энергетической точки зрения.

На рис. 4 показаны распределения градиентов давлений в камерах различных диаметров при $\mu=0.1$. По положению и форме фронта волны хорошо видно, что распространение волны в широких камерах $(\delta=3$ и 6) существенно отличается от случая приведенной камеры $(\delta=1)$. Во-первых, в широких камерах волны в ядре потока быстрее отражаются от задней стенки камеры в силу более короткого расстояния по оси. Вовторых, из-за наличия радиальной составляющей волны гораздо сильнее рассеиваются. Последнее обстоятельство способствует выравниванию параметров в резервуapaх широких камер.

\section{Влияние сжатия воздуха перед снарядом на выходную скорость}

В табл. 2 приведены результаты сравнения расчетных значений дульной скорости снаряда для указанных девяти случаев, полученных на одномерных и двумер-
Таблица 2. Результаты расчета дульной скорости $(\mathrm{m} / \mathrm{s})$ в зависимости от степени уширения камеры и массы метаемого тела

\begin{tabular}{c|c|c|c|c|c|c}
\hline \multirow{2}{*}{$\delta$} & \multicolumn{2}{|c|}{$m=0.1$} & \multicolumn{2}{c|}{$m=1$} & \multicolumn{2}{c}{$\mu=10$} \\
\cline { 2 - 7 } & $v_{p, 1 D}$ & $v_{p, 2 D}$ & $v_{p, 1 D}$ & $v_{p, 2 D}$ & $v_{p, 1 D}$ & $v_{p, 2 D}$ \\
\hline 1 & 460.9 & 434.0 & 230.7 & 229.9 & 85.0 & 85.0 \\
3 & 534.9 & 487.0 & 245.5 & 241.2 & 85.4 & 85.3 \\
6 & 517.5 & 486.2 & 239.9 & 240.1 & 85.0 & 86.0
\end{tabular}

Таблица 3. Сравнение значений дульных скоростей, полученных на квазиодномерной модели с учетом сжатия воздуха перед снарядом по формуле (3), и осесимметричной модели

\begin{tabular}{c|c|c|c}
\hline$\delta$ & $v_{p, 1 D}, \mathrm{~m} / \mathrm{s}$ & $v_{p, 2 D}, \mathrm{~m} / \mathrm{s}$ & разница, \% \\
\hline 1 & 421.4 & 434.0 & 2.9 \\
3 & 489.9 & 487.0 & 0.6 \\
6 & 474 & 486.2 & 2.5
\end{tabular}

ных осесимметричных моделях. Из приведенных данных следует, что для малых $\mu$ результаты двумерных и одномерных расчетов отличаются довольно существенно (на 5-10\%). Это происходит из-за того, что на движение легких снарядов оказывает влияние сжатие воздуха перед ними. Поскольку в осесимметричной постановке эти процессы учитываются введением отдельной расчетной области с воздухом, скорость получается значительно меньше. В одномерном случае учет сжатия воздуха перед снарядом можно произвести двумя способами. Первый способ - введение еще одной расчетной области с сеткой перед снарядом. Такой способ требует постановки дополнительных граничных условий и за- 


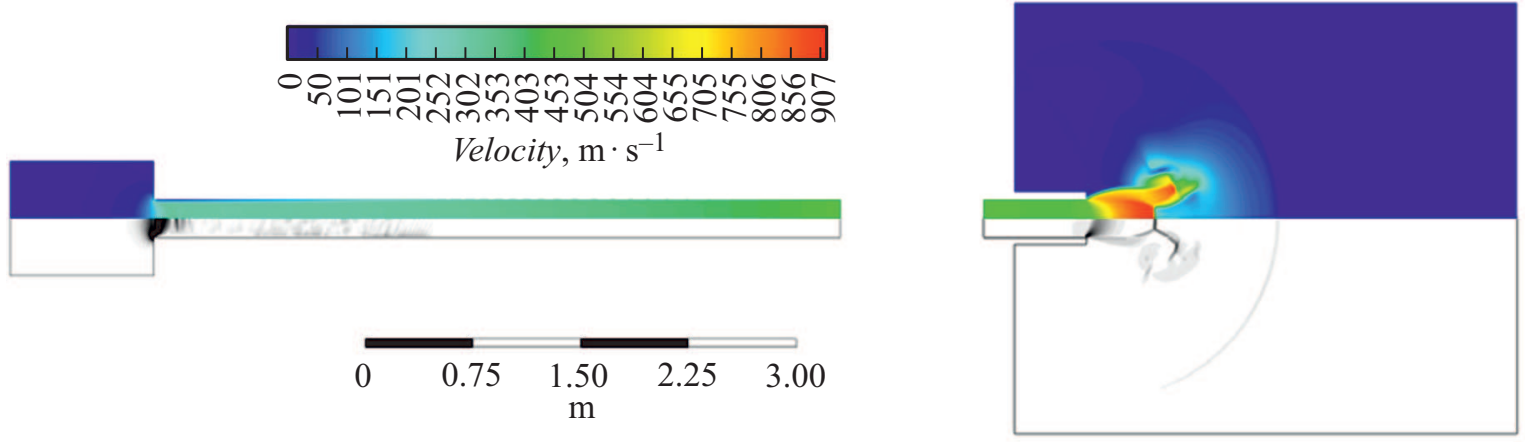

Рис. 5. Распределение скоростей (верхняя половина) и градиентов давления (нижняя половина) для случая $\mu=0.1$ в момент времени $t=1.5 \mathrm{~ms}$.

медляет время расчета примерно вдвое (в зависимости от подробности сетки, введенной в новой расчетной области). Второй способ - приближенный учет сжатия воздуха с использованием решения для вдвигающегося в газ с постоянной скоростью поршня [28]. Такой подход не требует введения дополнительных расчетных областей и поэтому удобнее первого. В этом случае давление перед снарядом в каждый момент времени вычисляется по формуле

$$
\begin{aligned}
p_{a}= & p_{0 a}\left(1+\frac{\gamma(\gamma+1)}{4}\left(\frac{v}{c_{0 a}}\right)^{2}\right. \\
& \left.+\gamma \frac{v}{c_{0 a}} \sqrt{1+\left(\frac{\gamma+1}{4} \frac{v}{c_{0 a}}\right)^{2}}\right) .
\end{aligned}
$$

Здесь $p_{0 a}$ - атмосферное давление в невозмущенном воздухе, $\gamma$ - показатель адиабаты воздуха, $c_{0 a}-$ скорость звука в невозмущенном воздухе.

Для оценки применимости такого способа в табл. 3 представлены результаты расчета скорости для случая $\mu=0.1$ с учетом сопротивления воздуха по формуле (3). Видно, что в этом случае разница не превышает $3 \%$.

Следует также обратить внимание на характерный излом на зависимостях скоростей от положения снаряда в окрестности точки $x=5 \mathrm{~m}$ в случае $\mu=0.1$ (кривые $1-3$ на рис. 3 ). Соответствующий этому положению снаряда момент изображен на рис. 5, где показаны распределения скоростей и градиентов давления. Выход ударной волны из ствола способствует образованию режима течения, при котором давление перед снарядом спадает интенсивнее, чем при движении ударной волны в трубе. Этот фактор способствует небольшому увеличению скорости.

\section{Оценка эффективности передачи энергии}

Проведем оценку энергетической эффективности передачи энергии в указанных случаях с помощью КПД,
Таблица 4. Результаты расчета КПД (\%) в зависимости от диаметра камеры и массы метаемого тела

\begin{tabular}{c|c|c|c}
\hline$\delta$ & $\mu=0.1$ & $\mu=1$ & $\mu=10$ \\
\hline 1 & 4.48 & 12.5 & 17.19 \\
3 & 5.64 & 13.8 & 17.32
\end{tabular}

равного отношению кинетической энергии снаряда на дульном срезе к начальной внутренней энергии сжатого газа:

$$
\eta=\frac{\gamma-1}{2} \frac{m v_{p}^{2}}{p_{0} W_{0}},
$$

где $p_{0}, W_{0}$ - начальные давление и объем сжатого газа соответственно.

В приведенных случаях и объем, и начальное давление газа всегда одинаковы, а изменяется только масса метаемого тела и его дульная скорость. В табл. 4 приведены значения КПД для $\delta=1$ и $\delta=3$, поскольку для $\delta=6$ значения не отличаются существенно. Значения скорости взяты из двумерных осесимметричных расчетов.

Таким образом, уширенные ресиверы позволяют увеличить КПД на 25\%. Следует отметить, что при малых $\mu$ и соответствующих им высоких скоростях, КПД установки достаточно мал - около 5\%. Это согласуется с выводом работы [22], согласно которому высокие значения КПД соответствуют низким скоростям и наоборот. Кроме того, согласно выводам работы [23], в рассматриваемом случае для реализации всех возможностей баллистической установки, необходимо сделать ее ствол в несколько раз длиннее.

\section{Заключение}

Проведено исследование влияния уширения газового ресивера и сжатия воздуха перед снарядом на баллистические характеристики лабораторных метательных установок на сжатом газе. Инструментами моделирования выступали квазиодномерная и двумерная осесиммет- 
ричная модели, валидированные на экспериментальных данных.

Полученные результаты продемонстрировали довольно существенное влияние уширения камеры на дульную скорость снаряда, что связано с тем, что на разгон снаряда на начальном этапе в значительной мере оказывают влияние волновые эффекты, с увеличением же уширения камеры уменьшается ее длина, что уменьшает путь отраженной от дна снаряда волны и позволяет ей догнать снаряд до его вылета из ствола. Отметим, что для камеры с наименьшим уширением и наибольшей длиной в заснарядном пространстве быстрее устанавливается линейный профиль скоростей, что является главным допущением в приближенном решении задачи с однородной деформацией. Более интенсивному обмену энергией между газом и разгоняемым телом также способствует ускорение газа при истечении из резервуара с уширением. Применение уширенных ресиверов при больших объемах газа позволяет задействовать в разгоне большую часть газа по сравнению с ресиверами приведенного диаметра. Это связано с большой разницей в длине хода волны в первом и втором случае. Уширенные ресиверы также позволяют повысить КПД установки приблизительно на $25 \%$.

Результаты расчетов также показали существенное влияние сжатия воздуха перед снарядом на его скорость, в особенности при сверхзвуковом его движении (для легких снарядов). В одномерной модели учет сжатия проведен приближенным способом, показавшим хорошие результаты при сравнении с двумерными расчетами. Также продемонстрирован эффект снижения давления перед снарядом за счет ускорения истечения газа при достижении фронтом ударной волны дульного среза. Этот эффект позволяет немного увеличить скорость тела перед вылетом.

Сравнение квазиодномерной и двумерной осесимметричной моделей показало, что использование первой может обеспечить достаточную точность при условии учета сжатия воздуха перед снарядом. Это обстоятельство довольно существенно в силу большой разницы в быстродействии этих двух моделей.

\section{Финансирование работы}

Работа выполнена при частичной финансовой поддержке Российского фонда фундаментальных исследований (проекты № 16-29-09596 офи-м и 16-38-00948_мол-а).

\section{Информация о вкладе авторов}

Двумерные расчеты в ANSYS Fluent проведены И.Е. Шестаковым.

\section{Конфликт интересов}

Авторы заявляют, что у них нет конфликта интересов.

\section{Список литературы}

[1] Bourne N.K. // J. Dyn. Behav. Mater. 2016. Vol. 2. N 1. P. 3342. DOI: $10.1007 / \mathrm{s} 40870-016-0055-5$

[2] Goveas S.G., Bourne N.K., Millett J.C.F. // AIP Conf. Proc. 2007. Vol. 955. N 2007. P. 525-528. DOI: $10.1063 / 1.2833130$

[3] Furmanski J., Carl P., Trujillo C.P., Martinez T.D., Gray III G.T., Brown E.N. // Polym. Test. 2012. Vol. 31. N 8. P. 1031-1037. DOI: 10.1016/j.polymertesting.2012.07.011

[4] Buslov E.P., Komarov I.S., Selivanov V.V., Titov V.A., Tovarnova N.A., Feldstein V.A. // Acta Astronaut. 2019. April. P. 1-8. DOI: 10.1016/j.actaastro.2019.04.046

[5] Duc L.D., Horák V., Vitek R., Kulish V. // 2017 International Conf. on Military Technologies (ICMT) (Brno, 2017). P. 1-6. DOI: 10.1109/MILTECHS.2017.7988720

[6] Григорьев В.В., Исаков С.Н., Петров Р.Л., Юркин С.В. // ЖТФ. 2006. Т. 76. Вып. 3. С. 75-80. [Grigor'ev V.V., Isakov S.N., Petrov R.L., Yurkin S.V. // Tech. Phys. 2006. Vol. 51. N 3. P. 367-372. DOI: 10.1134/S1063784206030121]

[7] Thunborg S., Ingram G.E., Graham R.A. // Rev. Sci. Instrum. 1964. Vol. 35. N 1. P. 11-14. DOI: $10.1063 / 1.1718685$

[8] Павленко А.В., Балабин С.И., Козелков О.Е., Казаков Д.Н. // ПТЭ. 2013. Т. 2013. Вып. 4. С. 122-124. [Pavlenko A.V., Balabin S.I., Kozelkov O.E., Kazakov D.N. // Instrum Exp Tech. 2013. Vol. 56. N 4. P. 482-484. DOI: $10.1134 / \mathrm{S} 0020441213040088]$

[9] Bourne N.K. // Meas. Sci. Technol. 2003. Vol. 14. N 3. P. $273-$ 278. DOI: $10.1088 / 0957-0233 / 14 / 3 / 304$

[10] Bourne N.K., Stevens G.S. // Rev. Sci. Instrum. 2001. Vol. 72. N 4. P. 2214-2218. DOI: $10.1063 / 1.1359192$

[11] Bourne N.K. // Rev. Sci. Instrum. 2004. Vol. 75. N 1. P. 253 258. DOI: $10.1063 / 1.1633988$

[12] Johnston A., Krishnamoorthy L.V. „A Numerical Simulation of Gas Gun Performance: Rep.“ in Defence Sci. Technol. Org. N DSTO-TN-080 (Edinburg, 2008).

[13] Gardiner P.A., Egawa Y., Watanabe K. // Mech. Eng. J. 2016. Vol. 3. N 6. P. 16-00273. DOI: $10.1299 /$ mej.16-00273

[14] Krishnamoorthy L., Stringer M., Taylor M.R.G., Kollias S. „Infrared countermeasure flare performance measurements using a gas gun“ in Proc. SPIE 5615, Technologies for Optical Countermeasures (29 December 2004). P. 54. DOI: $10.1117 / 12.583143$

[15] Fowles G.R., Duvall G.E., Asay J., Bellamy P., Feistmann F., Grady D., Michaels T., Mitchell R. // Rev. Sci. Instrum. 1970. Vol. 41. N 7. P. 984-996. DOI: $10.1063 / 1.1684739$

[16] Sheppard L.M. „Gas guns for aerodynamic testing at subsonic speeds" in Fifth Australian Conference on Hydraulics and Fluid Mechanics (Christianchurch, 1974). P. 514-521.

[17] Hutchings I.M., Winter R.E. // J. Phys. E. 1975. Vol. 8. N 2. P. 84-86. DOI: $10.1088 / 0022-3735 / 8 / 2 / 005$

[18] Hutchings I.M., Rochester M.C., Camus J.-J. // J. Phys. E. 1977. Vol. 10. N 5. P. 455-457. DOI: $10.1088 / 0022-3735 / 10 / 5 / 012$

[19] Porat Y., Gvishi M. // J. Phys. E. 1980. Vol. 13. N 5. P. 504 505. DOI: $10.1088 / 0022-3735 / 13 / 5 / 009$

[20] Дубовик А.В., Великовский Э.Т., Боболев В.К., Руссиян Е.К., Бражников Е.М., Земсков Н.А. // ФГВ. 1975. Вып. 1. С. 108-112. [Dubovik A.V., Velikovskii É.T., Bobolev V.K., Russiyan E.K., Brazhnikov E.M., Zemskov N.A. // Combust Explos Shock Waves. 1975. Vol. 11. N 1. P. 94-97. DOI: 10.1007/BF00742864] 
[21] Brown J.R., Chappell P.J.C., Egglestone G.T., Gellert E.P. // J. Phys. E. 1989. Vol. 22. N 9. P. 771-774.

DOI: $10.1088 / 0022-3735 / 22 / 9 / 016$

[22] Быков Н.В. // ПМТФ. 2019. Т. 60. № 3. С. 29-37. [Bykov N.V. // J. Appl. Mech. Tech. Phys. 2019. Vol. 60. N 3. P. 424-431. DOI: 10.1134/S0021894419030039]

[23] Быков Н.В. // Инженерный журнал наука и инновации. 2019. № 2(86). http://engjournal.ru/catalog/mech/ mlgp/1852.html [Bykov N.V. Engineer. J.: Sci. Innovation. 2019. N 2 (86). DOI: 10.18698/2308-6033-2019-2-1852]

[24] Булович С.В., Петров Р.Л. // Письма в ЖТФ. 2005. Т. 31. Вып. 16. C. 12-18. [Bulovich S.V., Petrov R.L. // Tech. Phys. Lett. 2005. Vol. 31. N 8. P. 682-684. DOI: 10.1134/1.2035365]

[25] Moradi A., Ahmadikia H. // Adv. Theor. Appl. Mech. 2011. Vol. 4. N 3. P. 101-111.

[26] Moradi A., Khodadadiyan S. // Int. J. Mech. Aerospace, Ind. Mechatron. Manuf. Eng. 2011. Vol. 5. № 2. P. 1081-1085. DOI: doi.org/10.5281/zenodo.1331541

[27] Хоменко Ю.П., Ищенко А.Н., Касимов В.З. Математическое моделирование внутрибаллистических процессов в ствольных системах. Новосибирск: Изд-во СО РАН, 1999. $256 \mathrm{c}$.

[28] Ландау Л.Д., Лившии, Е.М. Теоретическая физика. Т. VI. Гидродинамика. 5-е изд. М.: Физматлит, $2006.736 \mathrm{c}$. [Landau L.D., Lifshitz E.M. Fluid Mechanics. 2-nd ed. Oxford: Pergamon Press, 1987. 539 p.] 